\title{
Devenir docteur. Une formation par la recherche, véritable tremplin vers la construction de son identité professionnelle et personnelle
}

Becoming a doctor. Training through research as a springboard for the construction of a professional and personal identity

Olivier Maurin

\section{CpenEdition}

Journals

Édition électronique

URL : https://journals.openedition.org/essais/10865

DOI : 10.4000/essais. 10865

ISSN : 2276-0970

Éditeur

École doctorale Montaigne Humanités

Édition imprimée

Date de publication : 15 avril 2022

ISBN : 979-10-970024-00-0

ISSN : $2417-4211$

\section{Référence électronique}

Olivier Maurin, « Devenir docteur. Une formation par la recherche, véritable tremplin vers la construction de son identité professionnelle et personnelle », Essais [En ligne], Hors-série 7 | 2022, mis en ligne le 01 février 2022, consulté le 19 janvier 2023. URL : http://journals.openedition.org/essais/ 10865 ; DOI : https://doi.org/10.4000/essais.10865

Ce document a été généré automatiquement le 19 janvier 2023.

Tous droits réservés 


\section{Devenir docteur. Une formation par la recherche, véritable tremplin vers la construction de son identité professionnelle et personnelle}

Becoming a doctor. Training through research as a springboard for the construction of a professional and personal identity

\section{Olivier Maurin}

1 La période entre 2000 et 2012 a vu une progression significative du nombre de nouveaux docteurs en Europe $(+56 \%)$. La formation par la recherche reste une expérience unique et formatrice sur les plans personnels et professionnels.

Chaque thèse est en effet unique, non seulement en raison du sujet et de son domaine de spécialisation, mais également par le parcours personnel et académique du candidat. Une thèse de doctorat est par définition jalonnée de défis, de joies, de doutes, de remises en question. En cela, elle est une aventure intérieure (réflexion) et extérieure (émotionnelle) qui développe des compétences académiques et qui teste votre abnégation sur le plan personnel. Pour ma part, elle est donc une expérience au sens empiriste $^{1} \mathrm{du}$ terme, selon le concept théorisé par l'humaniste et philosophe anglais John Locke (1632-1704).

2 Les émotions intérieures et extérieures que cette aventure a pu susciter en moi ont eu pour conséquence de forger mes identités personnelles et professionnelles. Il convient ici de s'attarder sur le concept d'identité tant celui-ci est polymorphe et actuellement connoté. Pour définir pleinement les concepts d'identités personnelles et professionnelles, je souhaite m'appuyer sur l'approche sociologique pour renforcer celle de la philosophie :

3 Ainsi, les identités personnelles et professionnelles sont, " pour les sociologues, la mise en perspective de deux séries de données ou d'approches : des éléments biographiques issus d'approches longitudinales ou rétrospectives permettant de saisir les trajectoires, 
itinéraires et cheminements à travers lesquels les individus construisent leur appartenance et leur vision du monde et d'eux-mêmes ; des éléments structurels issus d'observations et d'analyses des pratiques et politiques d'emploi, de travail et de formation. C'est au croisement de ces deux perspectives que la notion d'identité, en tant que processus dynamique, prend tout son sens $»{ }^{2}$

Dans la lignée de cet empirisme (source de connaissances), défini par John Locke au XVIIe siècle, les savoirs, les savoir-faire et les savoir-être que nous utilisons à la fois pour interagir dans les contextes personnels et professionnels sont effectivement indissociable et de nature dynamique, c'est-à-dire en constante évolution grâce à la posture réflexive que nous adoptons. L'identité est donc un concept en mouvement qui se construit et se développe constamment au gré des contextes rencontrés pendant et après la formation à la recherche, aussi bien dans les environnements de formation, personnels ou professionnels.

5 Le doctorat mérite donc d'être vécu, car il engendre cette confrontation des éléments biographiques et structurels (d'abord dans le cadre de la formation, puis personnel, car les apprentissages modifient notre manière de percevoir le monde, et enfin professionnel lorsqu'il vient le temps de mettre en pratique les compétences acquises lors de sa thèse de doctorat) qui développe les identités personnelles et professionnelles. Les capacités d'introspection et de réflexivité que le doctorat demande sont, à mon sens, la plus grande valeur ajoutée des docteurs, toutes disciplines confondues, parce que l'introspection et la réflexivité sont les sources du développement des savoirs et elles permettent de s'adapter à des contextes professionnels multiples.

\section{Un sujet de thèse qui est le reflet d'un parcours de formation européen}

6 La thèse que j'ai pu mener de 2010 à 1016 s'intitule La Hongrie et les Pays-Bas méridionaux ${ }^{4}$ durant la guerre de Succession d'Espagne: les ambitions de la diplomatie française. Elle représente un important travail de recherche au sein de plusieurs centres d'archives à travers l'Europe (Belgique, France, Hongrie), de consultation des auteurs de référence exerçant dans tous les territoires concernés par le sujet de la thèse de doctorat, mais aussi d'écriture ${ }^{5}$. La construction de mon sujet est une émanation de mon parcours universitaire réalisé en Belgique, en France et en Hongrie. Il me permet d'intégrer les expériences et les points de vue des historiographies nationales que j'ai pu acquérir tout au long de ma formation. Ce sujet de recherche a eu l'ambition de faire la synthèse de mes expériences personnelles et de formation en France et à l'étranger.

7 J'ai eu la possibilité de participer au programme d'échange européen Erasmus en intégrant l'Université ELTE Budapest, lors du premier semestre de l'année 2008, afin de me familiariser avec les dynamiques culturelles et territoriales de mon espace d'étude. C'est un des moments fondateurs de la construction de mon identité académique et historienne. En effet, cette expérience m’a donné la possibilité de développer une grande autonomie dans mes recherches et de comprendre que je devais arpenter l'espace géographique étudié pour saisir toutes ses spécificités culturelles. Je revenais à Bordeaux en master 2 pour obtenir ce diplôme en juin 2009 en me donnant la possibilité d'envisager un doctorat, si mes recherches étaient validées. 
Cependant, je savais que mes études allaient probablement me destiner à l'enseignement secondaire ou supérieur ${ }^{6}$, et je devais d'abord m'assurer un avenir professionnel. Après mon expérience en Hongrie, j'avais pleinement intégré le fait que l'Union européenne offrait de multiples possibilités sur les plans professionnels et académiques. Il n'était donc pas obligatoire de se cantonner aux possibilités nationales, notamment à la perspective riche, mais toujours incertaine, de concours comme le CAPES ou l'agrégation'.

Pour obtenir un diplôme d'enseignement axé sur la didactique de l'histoire et la pédagogie, réalisable en une année, sans concours, je faisais le choix de poser ma candidature en Belgique à l'Université Catholique de Louvain-la-Neuve, afin de suivre une Agrégation de l'Enseignement Secondaire Supérieur (AESS) ${ }^{8}$. Cette formation, très concrète sur le plan pédagogique, comprenait des cours théoriques et trois stages actifs au sein de l'équivalent des lycées. En 2010, j'obtenais cette agrégation qui m'assurait un débouché professionnel. Cette condition était pour moi indispensable avant de débuter une thèse de doctorat. De plus, mon expérience en Belgique me permettait de me familiariser avec le second espace d'étude de ma thèse, à savoir les Pays-Bas méridionaux, un ancien ensemble territorial dont la situation correspond à celle de la Belgique actuelle et qui est un théâtre d'opérations central dans le conflit (Guerre de Succession d'Espagne) que j'avais étudié lors de mes années de master en France et en Hongrie.

10 Je retournais alors à l'Université de Bordeaux pour réaliser un doctorat en histoire moderne et contemporaine dans le cadre d'une cotutelle internationale sous la direction de directeurs de thèses spécialistes de ces questions dans leurs universités respectives (Université Bordeaux-Montaigne ${ }^{9}$ et Université Saint-Louis Bruxelles ${ }^{10}$ ). Je fus intégré au CEMMC ${ }^{11}$, ainsi qu'à l'Ecole Doctorale Montaigne Humanités.

\section{Le défi de financer ses recherches : l'opportunité d'acquérir de l'expérience et de consolider ses identités personnelles et professionnelles}

11 Six années furent nécessaires à sa réalisation. Son approche pluridisciplinaire, internationale et comparative n'explique pas entièrement la durée de sa réalisation. En 2011, malgré les efforts déployés par mon directeur de thèse pour défendre mon sujet de thèse devant le jury pour l'obtention des bourses doctorales de l'Université, je ne pus l'obtenir. Cependant, il put me faire bénéficier de la bourse du Comité pour l'Histoire de la Poste à hauteur de 5000 euros (somme versée en trois fois). Parallèlement, entre 2012 et 2014, j'avais la chance d'obtenir des heures de cours à dispenser aux étudiants en licence 1, 2 et 3, dans le cadre de travaux dirigés. En plus de ces heures de chargé de cours qui me ravissaient, je restais trois ans assistant d'éducation au lycée François Magendie de Bordeaux. Mon directeur de thèse français me permettait, durant cette période, de participer à plusieurs colloques organisés par l'école doctorale, le CEMMC ou le Comité pour l'Histoire de la Poste et de publier plusieurs fois entre 2010 et 2014, en France et en Hongrie. Ces expériences me donnèrent la chance de m'insérer concrètement dans les activités de recherche, de confronter mes premières conclusions à l'avis de spécialistes reconnus, de faire mes premières armes dans l'Enseignement Supérieur et de comprendre que je voulais 
évoluer professionnellement auprès de ce public. Grâce à mes activités de recherche lors de la thèse de doctorat, je pouvais ainsi acquérir la certitude que mon identité professionnelle $\mathrm{du}$ moment pouvait pleinement correspondre avec les missions inhérentes à un enseignant-chercheur.

12 En attendant, malgré l'aide précieuse de ma famille, ces financements internes restaient insuffisants pour me consacrer totalement à mes recherches et me déplacer dans l'ensemble des centres d'archives, je devais donc trouver d'autres sources de financements. Jusqu'à la soutenance, les expériences professionnelles dans l'enseignement français et belge furent multiples (niveaux, contextes, aptitudes à mettre en œuvre dans des contextes allant de la Zone d'Éducation Prioritaire au collège de Maubeuge, au lycée français de Bruxelles, en passant par une classe-hôpital). Elles me confortèrent dans mes ambitions académiques, tout en me permettant de développer mes compétences personnelles d'adaptation à divers environnements professionnels. L'ensemble des expériences personnelles et professionnelles vécues depuis le commencement de mon doctorat en 2010 allaient m'être extrêmement utiles dans le poste que j'obtenais dans l'Enseignement Supérieur belge au mois de novembre 2015.

13 À cette date, je devenais Maître-assistant en histoire et didactique de l'histoire au sein du département pédagogique de la Haute-Ecole Provinciale de Hainaut-Condorcet ${ }^{12}$ à Mons. Cette institution forme notamment des étudiants au métier de professeur dans le cadre d'un parcours professionnalisant sur trois ans. J'intégrais le cursus Agrégation de l'Enseignement Secondaire Inférieur (AESI) ${ }^{13}$, spécialité Sciences humaines. Cette formation regroupe des disciplines comme l'histoire, la géographie et les sciences sociales, la pédagogie, ainsi que la didactique de ces disciplines.

14 Sur les trois années, les missions qui me sont confiées correspondent à un enseignement historique généraliste ${ }^{14}$ et didactique de l'histoire. Pour chaque thématique, des supports de cours sont à créer avec des PowerPoint détaillés et des syllabus (mémoires) parfois de plus de 100 pages ${ }^{15}$, mettant à contribution les savoirfaire développés dans le cadre de la recherche : synthétiser, référencer, communiquer à l'écrit ses résultats de manière structurée. J'encadre également les recherches de mes étudiants lors de courts mémoires de fin d'études. À une année du terme de la thèse, son financement était assuré et la perspective d'un emploi durable, correspondant à mes aptitudes personnelles et à mes ambitions professionnelles, s'annonçait dans l'Enseignement Supérieur belge.

15 Rétrospectivement, cette recherche de financements fut une épreuve, mais aussi une chance, car elle me permettait d'exercer des fonctions dans l'enseignement secondaire et supérieur qui me permirent de développer de nouvelles qualités personnelles et académiques liées à ces expériences, que je peux mettre actuellement en œuvre auprès des étudiants.

16 Je renforçais par la suite ces compétences avec une formation universitaire d'une année (UCL) consacrée au professorat dans l'Enseignement Supérieur, avec l'obtention en 2019, du Certificat d'Aptitude Pédagogique Approprié à l'Enseignement Supérieur (CAPAES). Cette expérience de 2019 m'ancrait professionnellement en Belgique.

17 Par conséquent, la plus grande partie de mes compétences professionnelles restent de nature historiques et didactiques. Elles sont largement issues des compétences issues de la thèse au travers de la mise en perspective de ma culture historique, de mes 
méthodes de recherche dans le cadre d'une chronologie bornée à la guerre de Succession d'Espagne (1700-1714) ${ }^{16}$ d'une part, et aux espaces géographiques occupés par les deux provinces périphériques habsbourgeoises : les Pays-Bas méridionaux et la Hongrie d'autre part, sans oublier l'analyse des actions menées par les agents du corps diplomatique français et étranger servant ou combattant les ambitions dynastiques de Louis XIV sur ses territoires.

Mes recherches ont cherché à utiliser mes connaissances relatives aux espaces étudiés (territoires, centres d'archives, histoires nationales) pour mettre en évidence une guerre de l'ombre, encore très actuelle, celle de la diplomatie et du renseignement ${ }^{17}$. Tout en me faisant arpenter les routes européennes que j'avais eu l'occasion de découvrir durant mon cursus, cette analyse globale a donc eu pour ambition de mettre à profit les expériences personnelles et professionnelles que j'ai eu la chance de vivre durant mon cursus en essayant de faire la synthèse des différentes visions historiques que j'ai pu rencontrer en Belgique, en France ou en Hongrie.

\section{Une méthodologie de recherche construite tout au long de mon parcours de formation}

19 Pour construire ma méthodologie de recherche, je me suis appuyé sur mon parcours de formation en Belgique, en France et en Hongrie en adoptant une vision résolument européenne, pluridisciplinaire et globalisée, trois postures qui expliquent l'amplitude thématique et chronologique de ce travail qui est directement inspiré du courant historiographique de l'histoire des mentalités. Ce courant vise à remettre en perspective les champs de recherche en définissant le plus précisément possible le contexte de déroulement de l'étude, cela sur la base de sources manuscrites, imprimées et de seconde main, mais aussi de documents issus d'autres disciplines pour compléter les points de vue historiques ${ }^{18}$.

20 Cette méthodologie générale s'inspire des enseignements de mes directeurs de thèse et d'une conférence de l'historien canadien Gregory Hanlon organisée en 2010 à l'Université par l'École doctorale et le CEMMC. Il y fit l'éloge de la pluridisciplinarité pour remettre pleinement en contexte un sujet d'étude, afin de mieux le comprendre et pouvoir l'analyser, puis le critiquer le plus précisément possible.

21 J'ai donc utilisé cette approche pour affiner ma méthode de recherche et mettre systématiquement en contexte les postures et les stratégies mises en œuvre en Hongrie, en France et aux Pays-Bas méridionaux par les protagonistes de ma thèse durant la guerre de Succession d'Espagne. Outre l'exposition des faits, la finalité était donc de savoir si les acteurs de mon étude se plaçaient en rupture ou dans la lignée de la tradition (visions "nationales»), et si leurs pratiques et leurs analyses étaient bien adaptées au contexte des théâtres d'opération militaire (vision locale), diplomatique ou politique (vision étatique) au travers de problématiques liées à l'identité des populations locales, la nature géographique, sociale ou culturelle du territoire, la situation militaire et stratégique du moment, la nature des politiques menées par la France à l'étranger vis-à-vis de ses ennemis ou de ses alliés.

22 Ce travail de mise en contexte est à mon sens une des premières missions de l'historien, même s'il doit remonter loin dans le temps, car il permet d'éviter l'écueil de l'analyse des faits du passé avec notre regard d'aujourd'hui. Comme pour analyser un sujet de 
manière critique aujourd'hui, la compréhension du contexte est indispensable, car il permet ainsi de comprendre les mécanismes de pensée des protagonistes et donc leurs prises de décisions qui influencent le déroulement de l'histoire et les dynamiques qui sont à l'œuvre.

Cette mise en contexte systématique et cette approche critique sont les aspects les plus importants que j'enseigne aujourd'hui à mes étudiants. Mon parcours de formation en France, en Belgique et en Hongrie, dont la thèse de doctorat est l'aboutissement d'un point de vue historique, m'a donné la possibilité d'acquérir cette sensibilité et de l'intégrer dans mon activité professionnelle.

Pour exploiter pleinement cette sensibilité, dès le mémoire de master, il m'a semblé nécessaire de résider dans les territoires étudiés, afin de découvrir l'identité des populations, la nature des courants historiographiques locaux et de visualiser les environnements dans lesquels se déroule mon étude (bien qu'ils aient changé depuis). C'est ainsi que dès 2008, j'ai pu appréhender la Hongrie dans son ensemble avec le programme d'échange universitaire européen Erasmus. Cette expérience est fondamentale pour comprendre la démarche de mon étude et la vision que je développe à propos de la guerre d'Indépendance de Hongrie et de la nature de ses relations avec la France et avec les Pays-Bas méridionaux. J'ai reproduit cette expérience en Belgique à deux reprises, d'abord entre 2009 et 2010, puis de 2014 à aujourd'hui. Ces expériences ont eu pour effet de m'aider à construire mon sujet de recherche et de faciliter les analyses issues des dépouillements ${ }^{19}$ en essayant d'utiliser un regard propre à chaque territoire.

Durant ces années de recherches, j'ai donc pu affiner mon identité historienne (composée de mes expériences personnelles et professionnelles) sur la base de la méthode de recherche que je viens de décrire et cette approche critique contextualisée dans l'enseignement que je dispense aujourd'hui aux futurs professeurs d'histoire.

\section{Valorisation des compétences acquises lors de ma formation à la recherche dans ma vie personnelle et professionnelle}

Pour finir, la formation à la recherche permet donc de développer des compétences multiformes qui vont au-delà de la discipline. Elles sont autant d'empirismes (expériences générant des connaissances) qui m'ont permis de développer une grande capacité d'adaptation, un esprit critique et des valeurs comme celle du travail, de l'abnégation, du dépassement de soi, de l'ouverture d'esprit, de l'humilité, de la remise en question des méthodologies de recherche et des modes de pensée, qui sont aujourd'hui des atouts dans mon institution, mais qui sont souvent trop sous-estimés dans nos mondes contemporains (notamment pour les Sciences humaines), mis à part dans la recherche et l'éducation.

C'est grâce à cela que j'ai pu réaliser mon souhait de valoriser ma thèse de doctorat dans l'Enseignement Supérieur et de prendre mes fonctions à la HEPH-Condorcet, dont les missions se rapprochent de celles des INSPE ${ }^{20}$. Mon poste me permet de faire la synthèse de tous les enseignements que j'ai pu recevoir tout au long de mon cursus universitaire, notamment durant le doctorat (recherche). Je peux désormais les transmettre aux étudiants (synthèse, récoltes et analyses des données dans un contexte 
défini, communication écrite structurée, esprit critique, réflexivité, abnégation). J’ai fait le choix de partir à l'étranger pour me démarquer de la forte concurrence et pour valoriser une riche formation doctorale, car les institutions françaises n'ont pas toujours les moyens d'absorber le produit de leur formation, $d u$ fait du faible nombre de places à pourvoir dans les institutions de l'Enseignement Supérieur.

Aujourd'hui, les compétences acquises m'offrent aussi la possibilité de continuer à évoluer personnellement et professionnellement en tirant profit des empirismes (la crise sanitaire que nous traversons fut par exemple riche en enseignements). Tout d'abord avec la dispense de formations à des professeurs déjà diplômés, puis avec des missions transversales dans mon institution, au travers de la coordination de l'équipe professorale enseignant au sein de mon cursus de formation ( 20 professeurs) et mon entrée au conseil de département (2020), et enfin, avec la représentation des relations internationales au sein de mon cursus de formation. J'ai eu l'occasion de signer une convention Erasmus pour organiser la mobilité d'un de mes étudiants à l'INSPÉ Bordeaux en septembre 2020, clin d'œil à ma principale université de formation, Bordeaux-Montaigne.

Finalement, le poste que j'occupe aujourd'hui n'est que l'aboutissement d'un parcours quelque peu atypique, international, complet, et le reflet d'une certaine opiniâtreté à valoriser les compétences acquises dans le cadre de ma formation à la recherche. Cette dernière me permet, encore aujourd'hui, d'évoluer au sein de mon institution et de poursuivre la construction de mes identités personnelles et professionnelles en continuant à adopter une posture critique et réflexive vis-à-vis de mes propres compétences et expériences.

Enfin, les visions nationales influencent parfois encore trop le traitement des sujets de recherche. Néanmoins, les principales forces de cette formation à la recherche restent le développement de compétences (savoirs, savoir-faire et savoir-être) qui vont au-delà de la simple formation académique, puisqu'elles permettent à celui qui a la chance de la suivre d'évoluer personnellement tout au long de sa carrière professionnelle et de son existence grâce aux postures réflexives et critiques qu'elle engendre.

\section{NOTES}

1. L'empirisme est la connaissance qui est acquise au travers des expériences de vie (inhérentes à la thèse par exemple) grâce à la réflexivité qu'elle engendre. La première source de savoirs est dite extérieure, car elle provient des émotions ressenties face à une situation donnée, tandis que la seconde est intérieure, car elle a pour origine la réflexion. Cette introspection intérieure et extérieure issue d'une expérience favorise l'esprit critique et une remise en question permanente, source de connaissances.

2. Claude Dubar, «Identités collectives et individuelles dans le champ professionnel ", in Michel de Coster, François Pichault, Traité de sociologie du travail, Bruxelles, De Boeck Supérieur, 1998, p. 401.

3. La Grande Hongrie d'avant le traité de Trianon de 1920. 
4. En grande partie la Belgique (sauf la province Liège) et le Luxembourg actuels. En 1700, elles appartiennent à l'Espagne depuis 1556, puis deviennent autrichiennes en 1714 .

5. Ma thèse de doctorat en histoire moderne présente un volume de 765 pages de texte et 85 pages d'annexes. La norme se situe plutôt autour de 500 pages. Ce dépassement s'explique par l'ampleur du sujet à traiter et la nécessité d'intégrer les différents points de vue et contextes que j'ai pu rencontrer dans les sources, mais aussi dans mes formations universitaires en France, en Belgique et en Hongrie.

6. L'enseignement est le principal débouché professionnel en histoire en 2009 (année de l'obtention de mon master 2 en Histoire) comme en 2020. En effet, la majorité des étudiants qui obtiennent le diplôme de master 2 en histoire présentent ensuite les concours de l'enseignement, puis intègrent l'Éducation Nationale en France en qualité de professeur d'Histoire, de Géographie et d'Éducation Morale et Civique dans l'enseignement secondaire. L'issue incertaine puisque les taux de réussite pour le concours du CAPES externe en histoire étaient respectivement de 15,66\% en 2009 contre $21,66 \%$ en 2020 , selon «devenirenseignant.gouv.fr ». La proportion se réduit encore pour l'agrégation en histoire, avec un taux de réussite de 5,97\% en 2009 et $12,05 \%$ en 2020 , toujours selon « devenirenseignant.gouv.fr».

7. C'est statistiquement la norme en histoire. La majorité des étudiants qui obtiennent le diplôme de master 2 en histoire présentent ensuite les concours de l'enseignement, puis intègrent l'Éducation Nationale en France en qualité de professeur d'Histoire, de Géographie et d'Éducation Morale et Civique dans l'enseignement secondaire.

8. Jean-Louis Jadoulle, Faire apprendre l'Histoire : pratiques et fondements d'une didactique de l'Enquête en classe du secondaire, Namur, Érasme, 2015

9. François Cadilhon, Michel Figeac, Caroline Le Mao, La correspondance et les identités en Europe centrale (1648-1848), Paris, Honoré Champion, 2013. François Cadilhon, La Hongrie moderne: 1450-1850, Bordeaux, PUB, 2005.

10. Sébastien Dubois, L'invention de la Belgique. Genèse d'un État-nation, 1648-1830, Bruxelles, Racine, 2005.

11. CEMMC : Centre d'Étude des Mondes Moderne et Contemporain (EA 2958). Le sujet de ma thèse s'insère toujours dans l'axe numéro 1 de recherche du centre : «Pouvoirs : acteurs, espaces et représentations", ainsi que dans l'axe transversal : "Circulations et échanges en Europe Centrale et Orientale $\mathrm{XVI}^{e}-\mathrm{XXI}^{e}$ siècles». Ses approches pluridisciplinaires reflètent la formation que j'ai pu recevoir à l'École doctorale et à l'UFR d'Histoire.

12. La Haute-Ecole Provinciale de Hainaut - Condorcet (HEPH-Condorcet) résulte de la fusion de trois institutions provinciales d'Enseignement Supérieur (2009). Elle propose une cinquantaine de formations. La Haute-Ecole regroupe aujourd'hui 8000 étudiants, 700 membres du personnel sur 7 implantations.

13. En Belgique, l'Enseignement Secondaire Inférieur correspond aux classes de $5^{\mathrm{e}}, 4^{\mathrm{e}}$ et de 3e secondaire.

14. Tout d'abord l'Hominisation, la Mésopotamie, l'Égypte, la Grèce et Rome en première année, puis la civilisation islamique et les mondes chrétiens médiévaux en deuxième année, et enfin la Renaissance, l'Humanisme, le siècle des Lumières, les Révolutions libérales et l'Autocratie absolue d'Ancien Régime en troisième année.

15. Ces supports de cours représentent près de 2000 pages et slides. La consolidation de mon poste, de mes pratiques professorales et le CAPAES furent des freins à la reprise de mes activités de recherche en lien avec ma thèse, et notamment à son amélioration sur la base du rapport de soutenance.

16. À la mort du roi d'Espagne Charles II de Habsbourg le 1er novembre 1700, les Bourbons et les Habsbourg d'Autriche cherchent à accaparer le fabuleux héritage territorial de l'Espagne en Europe, mais aussi dans le monde, par l'intermédiaire de leurs candidats respectifs : Philippe, duc d'Anjou et petit-fils de Louis XIV contre l'archiduc Charles de Habsbourg (branche autrichienne), 
le second fils de l'Empereur Léopold Ier. Elle est considérée par certains historiens comme la première véritable guerre mondiale en raison de ses théâtres d'opérations en Amérique du Nord, aux Caraïbes et aux Indes.

17. Les courriers allant d'un bout à l'autre du Saint-Empire doivent coordonner les actions menées dans les deux périphéries habsbourgeoises depuis Versailles, et la confidentialité devient un enjeu pour les belligérants face au contre-espionnage. La quête du renseignement est ainsi une préoccupation croissante pour les cours européennes.

18. L'ensemble des sources consultées correspondent à un volume de plusieurs milliers de pages de sources manuscrites (notamment des lettres des agents diplomatiques et des rapports des ambassades), qui sont complétées par des études historiques publiées du XVIII à nos jours. Elles proviennent de tous les espaces européens concernés par mes recherches: Belgique, Hongrie, Pologne, Espagne, Turquie, France, Pays anglo-saxons. Ce complément était obligatoire, car mes activités professionnelles pour financer ma thèse ne me permettaient pas de me rendre systématiquement dans tous les centres d'archives en Europe (Budapest, Vienne, Cologne, Munich, Madrid). J'adaptais ainsi une partie de ma méthodologie à mon propre contexte, en exploitant la numérisation des sources manuscrites et imprimées.

19. Voir note de bas de page $n^{\circ} 16$ pour observer la nature et le volume de ces dépouillements

20. Institut National du Professorat et de l'Éducation qui dépend des Ministères de l'Éducation Nationale et de l'Enseignement Supérieur et de la Recherche.

\section{AUTEUR}

\section{OLIVIER MAURIN}

Haute-École Provinciale de Hainaut-Condorcet (Belgique) 